PROCEEDINGS OF THE

AMERICAN MATHEMATICAL SOCIETY

Volume 130 , Number 12, Pages 3495-3506

S 0002-9939(02)06483-3

Article electronically published on March 29, 2002

\title{
TRACE METHODS IN TWISTED GROUP ALGEBRAS, II
}

\author{
J. M. OSTERBURG AND D. S. PASSMAN \\ (Communicated by Lance W. Small)
}

\begin{abstract}
In this note, we continue our discussion of trace methods in twisted group algebras. Specifically, we obtain the twisted analog of Bass' theorem on the traces of idempotents in ordinary group algebras. Indeed, we show that with suitable normalization, the characteristic 0 trace values of an idempotent are all contained in a cyclotomic field. The proof is a variant of the original argument combined with a reduction to finitely presented groups.
\end{abstract}

\section{ACtions And traces}

Let $K^{t}[G]$ denote a twisted group algebra of a multiplicative group $G$ over a field $K$. Then $K^{t}[G]$ is an associative $K$-algebra with $K$-basis $\bar{G}=\{\bar{x} \mid x \in G\}$ and with multiplication defined distributively by $\bar{x} \cdot \bar{y}=\tau(x, y) \overline{x y}$ for all $x, y \in G$. Here $\tau: G \times G \rightarrow K^{\bullet}$ is the twisting function, and we know that the associative law in $K^{t}[G]$ is equivalent to $\tau$ being a 2-cocycle (see [3] $\left.\S 1.2\right]$ ). Furthermore, we can always assume that $\overline{1}$ is the identity element of $K^{t}[G]$. Unlike ordinary group algebras, twisted group algebras do not have a natural basis. Specifically, if $K^{t}[G]$ is given, then we say that $\bar{G} \rightarrow \widetilde{G}=\{\tilde{g} \mid g \in G\}$ is a diagonal change of basis, if $\tilde{g}=d_{g} \bar{g} \in K^{\bullet} \bar{g}$ for all $g \in G$. This procedure then changes $\tau$ by a factor which can be an arbitrary 2 -coboundary, but we do insist that $d_{1}=1$, so that $\tilde{1}=1=\overline{1}$.

Let $\operatorname{tr}: K^{t}[G] \rightarrow K$ be defined linearly by $\operatorname{tr} \bar{x}=0$ if $1 \neq x \in G$ and $\operatorname{tr} \overline{1}=1$. Then, for any element $\alpha \in K^{t}[G], \operatorname{tr} \alpha$ is the coefficient of the identity in $\alpha$, and it is easy to see that $\operatorname{tr} \alpha \beta=\operatorname{tr} \beta \alpha$ for all $\alpha, \beta \in K^{t}[G]$. In other words, $\operatorname{tr}: K^{t}[G] \rightarrow K$ is a trace map. As is well known, there are other trace maps defined on $K^{t}[G]$. To see this, it is convenient to first introduce some notation. If $x \in G$ and $\alpha \in K^{t}[G]$, then we define $\alpha^{x}=\alpha^{\bar{x}}=\bar{x}^{-1} \alpha \bar{x}$. Note that if $\bar{x}$ is replaced by any other element of $K^{\bullet} \bar{x}$, then $\alpha^{x}$ is unchanged. Thus, this conjugation is independent of a diagonal change of basis. Furthermore, from $\bar{x} \bar{y} \in K^{\bullet} \overline{x y}$, for $x, y \in G$, we see that $\left(\alpha^{x}\right)^{y}=\alpha^{x y}$. In this way, $G$ acts as automorphisms on $K^{t}[G]$.

Next, if $g \in G$, we let $\mathbb{C}_{G}^{t}(g)=\left\{x \in G \mid \bar{g}^{x}=\bar{g}\right\}$. Then $\mathbb{C}_{G}^{t}(g)$ is a subgroup of $G$. Furthermore, if $\bar{g}$ is replaced by any other element of $K^{\bullet} \bar{g}$, then this twisted centralizer remains unchanged, and hence it is also independent of a diagonal change

Received by the editors July 13, 2001.

2000 Mathematics Subject Classification. Primary 16S35.

The first author's research was supported by the Taft Committee of the University of Cincinnati. He would also like to thank the Mathematics Department of the University of Wisconsin for its hospitality. The second author's research was supported in part by NSF Grant DMS-9820271. 
of basis. Since $\bar{g}^{x}=\bar{x}^{-1} \bar{g} \bar{x} \in K^{\bullet} \overline{g^{x}}$ in general, it follows that $\mathbb{C}_{G}^{t}(g)$ is a subgroup of $\mathbb{C}_{G}(g)$. Furthermore, since ordinary and twisted conjugation are $G$ actions, we have $\mathbb{C}_{G}\left(g^{x}\right)=\mathbb{C}_{G}(g)^{x}$ and $\mathbb{C}_{G}^{t}\left(g^{x}\right)=\mathbb{C}_{G}^{t}(g)^{x}$.

We say that $g \in G$ is special if $\mathbb{C}_{G}^{t}(g)=\mathbb{C}_{G}(g)$. In view of the preceding comments, if $g$ is special, then so are all its conjugates $g^{x}$. Thus we can speak about special conjugacy classes, and we use $\mathfrak{K}_{g}$ to denote the class of $g$. Note that being special is not affected by a diagonal change of basis.

Lemma 1.1. Let $g \in G$.

(i) If $a$ and $b$ are relatively prime integers and if $g^{a}$ and $g^{b}$ are special, then $g$ is special.

(ii) If char $K=p>0$ and if $g^{p^{n}}$ is special, then $g$ is special.

Proof. (i) Let $x \in G$ commute with $g$ so that $\bar{x}^{-1} \bar{g} \bar{x}=\lambda \bar{g}$ for some $\lambda \in K^{\bullet}$. Then $x$ commutes with $g^{a}$ and, since $g^{a}$ is special, $\bar{x}$ and $\overline{g^{a}}$ commute. Furthermore, since $\bar{g}^{a} \in K \overline{g^{a}}$, we see that $\bar{x}$ and $\bar{g}^{a}$ commute. Hence $\lambda^{a} \bar{g}^{a}=\left(\bar{x}^{-1} \bar{g} \bar{x}\right)^{a}=\bar{x}^{-1} \bar{g}^{a} \bar{x}=\bar{g}^{a}$ and $\lambda^{a}=1$. Similarly, $\lambda^{b}=1$ and, since $a$ and $b$ are relatively prime integers, it follows that $\lambda=1$. Thus $\bar{x}$ and $\bar{g}$ commute, and $g$ is indeed special.

(ii) Let char $K=p>0$ and take $a=p^{n}$ in the above. Then $\lambda^{p^{n}}=1$, so $\lambda=1$ and therefore $\bar{x}$ and $\bar{g}$ commute, as required.

We say that the conjugacy class $\mathfrak{K}_{g}$ is consistent if for all $h, k \in \mathfrak{K}_{g}$, the equality $k=h^{y}$ implies that $\bar{k}=\bar{h}^{y}$. Note that consistency clearly requires that the conjugacy class be special. Conversely, any such special class can be made consistent via a diagonal change of basis that changes only those basis elements corresponding to members of the class. To see this, let $h \in \mathfrak{K}_{g}$. Then there exists $x$ in $G$ with $h=g^{x}$, and $x$ is unique up to a left factor in $\mathbb{C}_{G}(g)=\mathbb{C}_{G}^{t}(g)$. Thus $\bar{g}^{x}=\bar{x}^{-1} \bar{g} \bar{x}$ is uniquely determined by $h$, and we can make a diagonal change of basis so that $\bar{h}=\bar{g}^{x}$. Indeed, if we do this for all members of $\mathfrak{K}_{g}$, then $\mathfrak{K}_{g}$ becomes consistent since $h=g^{x}$ and $k=h^{y}$ imply that $k=g^{x y}$ and hence $\bar{k}=\bar{g}^{x y}=\left(\bar{g}^{x}\right)^{y}=\bar{h}^{y}$.

If $\mathfrak{K}_{g}$ is consistent, then we define $\operatorname{tr}_{\bar{g}}: K^{t}[G] \rightarrow K$ by

$$
\operatorname{tr}_{\bar{g}}: \sum_{x \in G} a_{x} \bar{x} \mapsto \sum_{x \in \mathfrak{K}_{g}} a_{x} .
$$

It is clear that $\operatorname{tr}_{\bar{g}}$ is a $K$-linear functional. Furthermore, we have

Lemma 1.2. If $\mathfrak{K}_{g}$ is consistent, then $\operatorname{tr}_{\bar{g}}$ is a trace map.

Proof. It suffices to show that if $x, y \in G$, then $\operatorname{tr}_{\bar{g}} \bar{x} \bar{y}=\operatorname{tr}_{\bar{g}} \bar{y} \bar{x}$. Since this is clear if neither $x y$ nor $y x$ is in $\mathfrak{K}_{g}$, we can assume that $x y=h \in \mathfrak{K}_{g}$. Then $\bar{x} \bar{y}=\lambda \bar{h}$ for some $\lambda \in K^{\bullet}$, and clearly $\lambda=\operatorname{tr}_{\bar{g}} \bar{x} \bar{y}$. Furthermore, if $k=h^{x}$, then consistency implies that $\bar{h}^{x}=\bar{k}$, and hence $\bar{y} \bar{x}=\bar{x}^{-1}(\bar{x} \bar{y}) \bar{x}=(\bar{x} \bar{y})^{x}=(\lambda \bar{h})^{x}=\lambda \bar{h}^{x}=\lambda \bar{k}$. Thus, $\operatorname{tr}_{\bar{g}} \bar{y} \bar{x}=\lambda=\operatorname{tr}_{\bar{g}} \bar{x} \bar{y}$, as required.

If $g$ is not special, then $\bar{g}$ vanishes on all trace maps. To see this, let $t: K^{t}[G] \rightarrow K$ be any trace, and choose $x \in \mathbb{C}_{G}(g) \backslash \mathbb{C}_{G}^{t}(g)$. Then $\bar{g}^{x}=\lambda \bar{g}$ with $\lambda \neq 1$ and, since $\bar{x}\left(\bar{x}^{-1} \bar{g}\right)-\left(\bar{x}^{-1} \bar{g}\right) \bar{x}=\bar{g}-\bar{g}^{x}=(1-\lambda) \bar{g}$, it follows that $t:(1-\lambda) \bar{g} \mapsto 0$ and hence that $t: \bar{g} \mapsto 0$. Note that if $g$ is special, then $\operatorname{tr}_{\bar{g}}$ depends upon $\bar{g}$ and not just on $g$. Indeed, suppose that $\mathfrak{K}_{g}$ is consistent and let $k \in K^{\bullet}$. If we make a diagonal change of basis by multiplying each $\overline{g^{x}}$ by $k$, then $\mathfrak{K}_{g}$ remains consistent, but gives rise to a new trace map $\operatorname{tr}_{k \bar{g}}$ satisfying 
Lemma 1.3. If $g$ is a special element of $G$ and if $k \in K^{\bullet}$, then $\operatorname{tr}_{k \bar{g}} \alpha=k^{-1} \cdot \operatorname{tr}_{\bar{g}} \alpha$ for all $\alpha \in K^{t}[G]$.

Thus the trace value of an element in $K^{t}[G]$ can be multiplied by an arbitrary element of $K^{\bullet}$ by merely making a diagonal change of basis. It follows that if we wish to speak about trace values, then we need to find canonical members of $K^{\bullet} \bar{g}$. To this end, let $g$ be a special element of $G$. If $g$ is not conjugate to any power $g^{n+1}$ with $n \geq 1$, then we say that $g$ has infinite conjugate order and write $c(g)=\infty$. If $g$ is conjugate to some power $g^{n+1}$ with $n \geq 1$, then we let $c(g)$ denote the minimal such $n$. Note that if $g$ has finite (ordinary) order $m$, then $g^{m}=1$, so $g^{m+1}=g$ is conjugate to $g$. Thus $c(g) \leq m=o(g)$. Furthermore, it is easy to see that the conjugate order is constant over a conjugacy class. Indeed, if $g^{c+1}$ is conjugate to $g$, then conjugating by $y$ shows that $\left(g^{y}\right)^{c+1}$ is conjugate to $g$ and hence to $g^{y}$.

Next, we define what it means for $\bar{g}$ to be normalized. First, if $c(g)=\infty$, then any choice for $\bar{g}$ is normalized. On the other hand, suppose that $c(g)=c$ is finite. Then $g^{c+1}$ is conjugate to $g$, say $g^{c+1}=g^{x}$ for some $x \in G$. It follows that $\lambda \bar{g}^{c+1}=\bar{g}^{x}$ for some $\lambda \in K^{\bullet}$, and as we indicated previously, $\bar{g}^{x}$ depends only upon $\bar{g}$ and $g^{x}$. In this case, we say that $\bar{g}$ is normalized if $\lambda=1$. Now suppose that $\bar{g}$ is given with $c(g)=c<\infty$ and let us make a diagonal change of basis by setting $\tilde{g}=k \bar{g}$ for some $k \in K^{\bullet}$. Then $\tilde{g}^{c+1}=k^{c+1} \bar{g}^{c+1}$ and $\tilde{g}^{x}=k \bar{g}^{x}$, so $\lambda \tilde{g}^{c+1}=k^{c+1} \lambda \bar{g}^{c+1}=k^{c+1} \bar{g}^{x}=k^{c} \tilde{g}^{x}$. Thus $\tilde{g}$ will be normalized precisely when $k^{c}=\lambda$, and we have proved

Lemma 1.4. Let $K$ be an algebraically closed field and let $g$ be special with $c(g)=$ $c<\infty$. Then, via a diagonal change of basis, changing only the basis element corresponding to $g$, we can make $\bar{g}$ normalized. Furthermore, $\bar{g}$ is then unique up to a factor of a $c^{\text {th }}$ root of unity in $K^{\bullet}$.

Note that $1 \in G$ is special and $1^{2}=1$, so $c(1)=1$. Thus $\overline{1}^{2}=\overline{1}$ implies that $\overline{1}=1$ is the unique normalized element associated with the identity of $G$. Of course, $\operatorname{tr}_{\overline{1}}: K^{t}[G] \rightarrow K$ is the usual trace map tr. We also note that if $\mathfrak{K}_{g}$ is consistent and if $\bar{g}$ is normalized, then $\bar{g}^{y}$ is normalized for all $y \in G$. This is immediate since $\bar{g}^{c+1}=\bar{g}^{x}$ implies that $\left(\bar{g}^{y}\right)^{c+1}=\bar{g}^{x y}=\left(\bar{g}^{y}\right)^{y^{-1} x y}$. Thus, we can speak about normalized classes. Now, the key fact about normalized elements is that they control all situations where $g^{n+1}$ is conjugate to $g$, not just the particular case when $n=c(g)$. Indeed, we have the following more general result, where we use $\sim$ to denote the fact that two group elements are conjugate.

Lemma 1.5. Suppose that $K^{t}[G]$ is given.

(i) Let $g, h \in G$ with $\mathfrak{K}_{g}$ and $\mathfrak{K}_{h}$ both normalized and consistent. If $g^{n} \sim h$ and $h^{m} \sim g$ for some positive integers $n, m$ with $n m>1$, then $c(g)=c(h)<\infty$ and $\operatorname{tr}_{\bar{h}} \bar{g}^{n}$ is a $c(g)^{\text {th }}$ root of unity.

(ii) Let $g \in G$ with $\mathfrak{K}_{g}$ normalized and consistent. If $g^{n} \sim g$ for some positive integer $n>1$, then $c(g)<\infty$ and $\operatorname{tr}_{\bar{g}} \bar{g}^{n}$ is a $c(g)^{\text {th }}$ root of unity.

Proof. (i) We have $g^{n m} \sim h^{m} \sim g$, so $c(g)<\infty$ since $n m>1$. Now, if $g^{k} \sim g$, then $h^{k} \sim g^{n k} \sim g^{n} \sim h$, and if $h^{k} \sim h$, then $g^{k} \sim h^{m k} \sim h^{m} \sim g$. Thus $c=c(h)=c(g)<\infty$, and since $\mathfrak{K}_{g}$ and $\mathfrak{K}_{h}$ are normalized, we have $\bar{g}^{c+1}=\bar{g}^{x}$ and $\bar{h}^{c+1}=\bar{h}^{y}$ for suitable $x, y \in G$. 
Finally, $\bar{g}^{n}=\lambda \bar{h}^{z}$ for some $\lambda \in K^{\bullet}$ and $z \in G$, and raising this equation to the $(c+1)^{\text {st }}$ power yields

$$
\left(\bar{g}^{n}\right)^{x}=\left(\bar{g}^{n}\right)^{c+1}=\lambda^{c+1}\left(\bar{h}^{c+1}\right)^{z}=\lambda^{c+1} \bar{h}^{y z} .
$$

Thus, $\lambda^{c+1}=\operatorname{tr}_{\bar{h}}\left(\bar{g}^{n}\right)^{x}=\operatorname{tr}_{\bar{h}} \bar{g}^{n}=\lambda$, and hence $\lambda^{c}=1$, as required.

(ii) Let $h=g$ and $m=1$ in the above, so that $g^{n} \sim h, h^{m} \sim g$ and $n m=n>1$. Then, by (i), $c(g)<\infty$ and $\operatorname{tr}_{\bar{g}} \bar{g}^{n}=\operatorname{tr}_{\bar{h}} \bar{g}^{n}$ is a $c(g)^{\text {th }}$ root of unity.

In the case of ordinary group rings, trace results in characteristic 0 are usually lifted from those of characteristic $p$ by means of a place argument. But in twisted group algebras, the set $\tau(G, G)=\{\tau(x, y) \mid x, y \in G\}$ can generate so large a subgroup of $K^{\bullet}$ as to effectively prevent the use of this technique. Indeed, even if $G$ is finitely generated, we could have $\langle\tau(G, G)\rangle=K^{\bullet}$ when $K$ is a countable field. Nevertheless, we can overcome this difficulty with the following trick that extends [4. Lemma 1.3]. Here, if $\alpha=\sum_{x} a_{x} \bar{x} \in K^{t}[G]$, then the support of $\alpha$ is the finite subset of $G$ given by $\operatorname{supp} \alpha=\left\{x \in G \mid a_{x} \neq 0\right\}$.

Lemma 1.6. Let $K^{t}[G]$ be a twisted group algebra, let $f(\zeta) \in K[\zeta]$ be a polynomial, and suppose that $\alpha \in K^{t}[G]$ satisfies $f(\alpha)=0$. Furthermore, let $\mathfrak{K}_{g}$ be a consistent, normalized conjugacy class in $G$. Then there exists a finitely presented group $H$, a twisted group algebra $K^{t}[H]$, an element $\beta \in K^{t}[H]$, and a consistent, normalized congugacy class $\mathfrak{K}_{h}$ in $H$, such that:

(i) $f(\beta)=0,|\operatorname{supp} \alpha|=|\operatorname{supp} \beta|$, and $\operatorname{tr}_{\bar{g}} \alpha=\operatorname{tr}_{\bar{h}} \beta$.

(ii) $c(g)=c(h)$ and $o(g)=o(h)$.

(iii) For any integer $n$, if $h^{n} \sim h$, then $g^{n} \sim g$, and if $h^{n}$ is conjugate to an element of $\operatorname{supp} \beta$, then $g^{n}$ is conjugate to an element of $\operatorname{supp} \alpha$.

Proof. Write $\alpha=\lambda_{1} \overline{g_{1}}+\lambda_{2} \overline{g_{2}}+\cdots+\lambda_{m} \overline{g_{m}} \in K^{t}[G]$ with $g_{1}, g_{2}, \ldots, g_{m}$ distinct elements of $G$, and with $\lambda_{1}, \lambda_{2}, \ldots, \lambda_{m}$ nonzero elements of $K$. We can assume that the numbering is so chosen that $g_{1}, g_{2}, \ldots, g_{r}$ are the elements of supp $\alpha$ that are conjugate to $g$. Then $\operatorname{tr}_{\bar{g}} \alpha=\lambda_{1}+\lambda_{2}+\cdots+\lambda_{r}$, and there exist elements $x_{i} \in G$ with $g^{x_{i}}=g_{i}$ for $i=1,2, \ldots, r$. If $c(g)=c<\infty$, fix $x \in G$ with $g^{x}=g^{c+1}$.

Let $F=F\left\langle z, z_{1}, \ldots, z_{m}, y_{1}, \ldots, y_{r}, y\right\rangle$ be a free group on $m+r+2$ generators and suppose that $\operatorname{deg} f(\zeta)=d$. Use $W$ to denote the finite set of semigroup words in $z_{1}, z_{2}, \ldots, z_{m}$ of degree $\leq d$ and define the equivalence relation $\approx$ on $W$ by $u \approx v$ if and only if $u\left(g_{1}, g_{2}, \ldots, g_{m}\right)=v\left(g_{1}, g_{2}, \ldots, g_{m}\right)$. Now let $N$ be the normal subgroup of $F$ generated by all expressions of the form $u v^{-1}$ with $u, v \in W$ and $u \approx v$, along with $z^{y_{i}} z_{i}^{-1}$ for $i=1,2, \ldots, r$, and the elements $z^{y} z^{-(c+1)}$ if $c=c(g)<\infty$ and $z^{o(g)}$ if $o(g)<\infty$. Then $N$ is finitely generated as a normal subgroup of $F$, so $H=F / N$ is a finitely presented group. Furthermore, $N$ maps to 1 under the natural homomorphism $F \rightarrow G$ given by $z \mapsto g, z_{i} \mapsto g_{i}, y_{i} \mapsto x_{i}$ and $y \mapsto x$, so we obtain a homomorphism $\theta: H \rightarrow G$ with $h=z N \mapsto g, h_{i}=z_{i} N \mapsto g_{i}$, $k_{i}=y_{i} N \mapsto x_{i}$ and $k=y N \mapsto x$

Next, observe that $\tau$ lifts to a 2-cocycle $\sigma: H \times H \rightarrow K^{\bullet}$ given by $\sigma(a, b)=$ $\tau(\theta(a), \theta(b))$ for all $a, b \in H$. This, in turn, allows us to define a twisted group algebra $K^{t}[H]$, using $\sigma$, and then $\theta$ clearly extends to an algebra homomorphism $\theta: K^{t}[H] \rightarrow K^{t}[G]$ via $\theta(\bar{a})=\overline{\theta(a)}$ for all $a \in H$. Indeed, $\theta$ is certainly $K$-linear 
and we have

$$
\begin{aligned}
\theta(\bar{a}) \theta(\bar{b}) & =\overline{\theta(a)} \cdot \overline{\theta(b)}=\tau(\theta(a), \theta(b)) \overline{\theta(a) \theta(b)} \\
& =\sigma(a, b) \overline{\theta(a b)}=\theta(\sigma(a, b) \overline{a b})=\theta(\bar{a} \bar{b})
\end{aligned}
$$

for all $a, b \in H$.

Define $\beta \in K^{t}[H]$ by $\beta=\lambda_{1} \overline{h_{1}}+\lambda_{2} \overline{h_{2}}+\cdots+\lambda_{m} \overline{h_{m}}$, so that $\theta(\beta)=\alpha$. Since $f(\zeta) \in K[\zeta]$ is a polynomial of degree $d$, it is clear that $f(\beta)$ can be written as

$$
f(\beta)=\sum_{w \in W} \mu_{w}^{\prime} \overline{w\left(h_{1}, h_{2}, \ldots, h_{m}\right)}
$$

for suitable $\mu_{w}^{\prime} \in K$. Note that this expression is not necessarily unique. Indeed, if $u, v \in W$ and if $u \approx v$, then the definition of $H$ implies that $u\left(h_{1}, h_{2}, \ldots, h_{m}\right)=$ $v\left(h_{1}, h_{2}, \ldots, h_{m}\right)$. Thus, we can replace each $w \in W$ in the above-displayed equation by a fixed representative of its equivalence class and obtain

$$
f(\beta)=\sum_{w \in W_{0}} \mu_{w} \overline{w\left(h_{1}, h_{2}, \ldots, h_{m}\right)},
$$

where $W_{0}$ is a set of representatives for the $\approx$ equivalence classes and $\mu_{w} \in K$. Now, via the homomorphism $\theta$, we have

$$
0=f(\alpha)=\theta(f(\beta))=\sum_{w \in W_{0}} \mu_{w} \overline{w\left(g_{1}, g_{2}, \ldots, g_{m}\right)} .
$$

But, by definition of $\approx$, it follows that the group elements $w\left(g_{1}, g_{2}, \ldots, g_{m}\right)$ with $w \in W_{0}$ are all distinct. Thus, since $f(\alpha)=0$, we must have $\mu_{w}=0$ for each $w \in W_{0}$, and hence $f(\beta)=0$. Note also that $g_{i} \neq g_{j}$ implies that $h_{i} \neq h_{j}$. Thus $|\operatorname{supp} \beta|=m=|\operatorname{supp} \alpha|$.

Finally, we consider the element $h=z N \in H$. To start with, since $\theta(h)=g$ and since $h^{o(g)}=1$ if $o(g)<\infty$, it follows that $o(h)=o(g)$. Next, if $a \in H$ commutes with $h$, then $\bar{h}^{a}=\lambda \bar{h}$ for some $\lambda \in K^{\bullet}$, and by applying $\theta: K^{t}[H] \rightarrow K^{t}[G]$, we obtain $\bar{g}^{\theta(a)}=\lambda \bar{g}$. In particular, $\theta(a) \in G$ commutes with the special element $g$, so $\lambda=1$. Thus $\bar{h}^{a}=\bar{h}$ and $\mathfrak{K}_{h}$ is a special class in $H$. This class is also consistent since, for any $b \in H$, applying $\theta$ to the equation $\bar{h}^{b}=\mu \overline{h^{b}}$ yields $\bar{g}^{\theta(b)}=\mu \overline{g^{\theta(b)}}$. But we know that $\mathfrak{K}_{g}$ is consistent, so $\mu=1$ and hence $\mathfrak{K}_{h}$ is consistent, as claimed.

Next suppose that $h^{s+1} \sim h$ for some integer $s \geq 1$. Then $\theta(h)=g$ implies that $g^{s+1} \sim g$ and hence that $s \geq c=c(g)$. On the other hand, if $c<\infty$, then $h^{c+1}=h^{k} \sim h$ by the definition of $k=y N \in H$. Thus we conclude that $c(h)=c(g)=c$. As a consequence, it also follows that $\bar{h}$ is normalized. Indeed, if $c<\infty$, then $\bar{h}^{c+1}=\rho \overline{h^{k}}=\rho \bar{h}^{k}$ for some $\rho \in K^{\bullet}$, and by applying $\theta$, we have $\bar{g}^{c+1}=\rho \bar{g}^{\theta(k)}$. Thus, since $\bar{g}$ is normalized and $c=c(g)$, we conclude that $\rho=1$ and hence that $\bar{h}$ is normalized. It now makes sense to consider the map $\operatorname{tr}_{\bar{h}}$ and in particular to compute $\operatorname{tr}_{\bar{h}} \beta$. Observe that $\operatorname{supp} \beta=\left\{h_{1}, h_{2}, \ldots, h_{m}\right\}$ and that by applying $\theta$ to the expression $h \sim h_{i}$, we get $g \sim g_{i}$ and hence $i \leq r$. On the other hand, by the definition of $k_{i}=y_{i} N$, we have $h_{i}=h^{k_{i}}$ for $i=1,2, \ldots, r$. Thus $\mathfrak{K}_{h} \cap\left\{h_{1}, h_{2}, \ldots, h_{m}\right\}=\left\{h_{1}, h_{2}, \ldots, h_{r}\right\}$, so $\operatorname{tr}_{\bar{h}} \beta=\lambda_{1}+\lambda_{2}+\cdots+\lambda_{r}=\operatorname{tr}_{\bar{g}} \alpha$, and parts (i) and (ii) are proved. In addition, if either $h^{n} \sim h$ or if $h^{n} \sim h_{j} \in \operatorname{supp} \beta$ for some integer $n$, then applying $\theta$ yields $g^{n} \sim g$ or $g^{n} \sim g_{j} \in \operatorname{supp} \alpha$, respectively, so (iii) follows.

The previous lemma could clearly be extended in a number of ways by merely adding additional subscripts. For example, we could deal with finitely many twisted 
group ring elements $\alpha_{1}, \alpha_{2}, \ldots, \alpha_{s}$ that satisfy various polynomials in one or more variables. In addition, we could consider finitely many trace functions determined by finitely many consistent, normalized classes. Fortunately, these more complicated versions are not needed here. As we see below, the reason for studying finitely presented groups in this context is that their possible twistings are not too large. The following observation extends [4 Lemma 1.4].

Let $\bar{G} \rightarrow \widetilde{G}$ be a diagonal change of basis, so that each $\bar{g} \in \bar{G}$ is replaced by $\tilde{g}=d_{g} \bar{g}$ for some $d_{g} \in K^{\bullet}$. We say that a subset $X \subseteq G$ is not affected by this basis change if $\tilde{x}=\bar{x}$ for all $x \in X$ or equivalently if $d_{x}=1$ for all such $x$. Note that if $\mathfrak{K}_{h}$ is a consistent class in $K^{t}[G]$ and if $\mathfrak{K}_{h}$ is not affected by the diagonal change of basis, then $\mathfrak{K}_{h}$ remains consistent and determines the same trace function. In other words, $\operatorname{tr}_{\tilde{h}}=\operatorname{tr}_{\bar{h}}$, so this trace function is also not affected by the basis change.

Lemma 1.7. Let $K^{t}[G]$ be a twisted group ring with $G$ a finitely presented group, and let $\mathfrak{K}_{g_{1}}, \mathfrak{K}_{g_{2}}, \ldots, \mathfrak{K}_{g_{s}}$ be finitely many consistent classes. Then there exists a diagonal change of basis, not affecting any of these finitely many classes or their associated trace functions, such that if $\tau: G \times G \rightarrow K^{\bullet}$ is the new twisting, then $\tau(G, G)$ is contained in a finitely generated subgroup of $K^{\bullet}$.

Proof. Extend the set $\left\{g_{1}, g_{2}, \ldots, g_{s}\right\}$ to a finite generating set $\left\{g_{1}, g_{2}, \ldots, g_{n}\right\}$ for $G$, and let $K^{\bullet} \bar{G}$ be the group of trivial units in $K^{t}[G]$. Then the map $\nu: K^{\bullet} \bar{G} \rightarrow G$ given by $k \bar{g} \mapsto g$ is an epimorphism. In particular, if $\mathcal{G}$ is the subgroup of $K^{\bullet} \bar{G}$ generated by $\overline{g_{1}}, \overline{g_{2}}, \ldots, \overline{g_{n}}$, then $\mathcal{G}$ is finitely generated and $\nu: \mathcal{G} \rightarrow G$ is onto. Of course, the kernel here is $\mathcal{G} \cap K^{\bullet} \triangleleft \mathcal{G}$ and, since $G$ is finitely presented, [3, Lemma 12.3.12(ii)] implies that $\mathcal{G} \cap K^{\bullet}$ is finitely generated as a normal subgroup of $\mathcal{G}$. But $\mathcal{G} \cap K^{\bullet}$ is central in $\mathcal{G}$, so it is necessarily a finitely generated abelian group.

Now observe that for any $1 \leq i \leq s$ and any $x \in G$, we have $\overline{g_{i}} \in \mathcal{G}$ and $\lambda \bar{x} \in \mathcal{G}$ for some $\lambda \in K^{\bullet}$. Thus since $\mathfrak{K}_{g_{i}}$ is consistent, $\overline{g_{i}^{x}}={\overline{g_{i}}}^{x}=(\lambda \bar{x})^{-1} \overline{g_{i}}(\lambda \bar{x}) \in \mathcal{G}$. Finally, let $\widetilde{G}$ be a transversal for $\mathcal{G} \cap K^{\bullet}$ in $\mathcal{G}$ chosen to contain all $\overline{g_{i}^{x}}$ and the identity element. Then $\widetilde{G}$ is clearly a new basis for $K^{t}[G]$ and $\bar{G} \rightarrow \widetilde{G}$ is a diagonal change of basis not affecting any of the finitely many classes $\mathfrak{K}_{g_{i}}$. Furthermore, if $\tau$ is the new twisting function, then $\tau(G, G) \subseteq \mathcal{G} \cap K^{\bullet}$, so the result follows.

Finally, if $R$ is a subring of the field $K$ containing $\tau(G, G)$ as a set of units, then the $R$-linear span of $\bar{G}$ is clearly a subring of $K^{t}[G]$ which we denote by $R^{t}[G]$. Note that each $\bar{x}$ is invertible in $R^{t}[G]$ since $\bar{x} \cdot \overline{x^{-1}}=\tau\left(x, x^{-1}\right)$ and $\overline{x^{-1}} \cdot \bar{x}=\tau\left(x^{-1}, x\right)$ are both units in $R$.

Lemma 1.8. Let $R$ be a subring of the field $K$ containing $\tau(G, G)$ as a set of units, and let $\theta: R \rightarrow F$ be a homomorphism to the field $F$. Then there exists a twisted group algebra $F^{t}[G]$ and a ring homomorphism $\theta: R^{t}[G] \rightarrow F^{t}[G]$ given by $\theta: \sum a_{x} \bar{x} \mapsto \sum \theta\left(a_{x}\right) \bar{x}$. Furthermore, if $\mathfrak{K}_{g}$ is a consistent, normalized class in $K^{t}[G]$, then $\mathfrak{K}_{g}$ is also a consistent, normalized class in $F^{t}[G]$, and we have $\operatorname{tr}_{\bar{g}} \theta(\alpha)=\theta\left(\operatorname{tr}_{\bar{g}} \alpha\right)$ for all $\alpha \in R^{t}[G]$.

Proof. Define a twisting function $\sigma: G \times G \rightarrow F^{\bullet}$ by $\sigma(x, y)=\theta(\tau(x, y))$ for all elements $x, y \in G$. Then $\sigma$ is clearly a 2-cocycle since $\tau(G, G)$ consists of units of $R$, and hence it determines a twisted group algebra $F^{t}[G]$. Furthermore, the map $\theta: R^{t}[G] \rightarrow F^{t}[G]$ described above is then a ring homomorphism since it is clearly additive and since, for all $x, y \in G$, we have

$$
\theta(\bar{x}) \theta(\bar{y})=\bar{x} \bar{y}=\sigma(x, y) \overline{x y}=\theta(\tau(x, y)) \overline{x y}=\theta(\tau(x, y) \overline{x y})=\theta(\bar{x} \bar{y}) .
$$


This also implies that $\theta$ commutes with the action of $G$, so that $\theta\left(\alpha^{x}\right)=\theta(\alpha)^{x}$ for all $\alpha \in R^{t}[G]$ and $x \in G$. With this, it follows that $\mathfrak{K}_{g}$ is consistent and normalized in $F^{t}[G]$, and then that $\operatorname{tr}_{\bar{g}} \theta(\alpha)=\theta\left(\operatorname{tr}_{\bar{g}} \alpha\right)$ for all $\alpha \in R^{t}[G]$.

\section{Traces of idempotents}

We now offer suitably modified versions of the arguments in [1] and its presentation in [5, $\S 1.1]$. We begin with the characteristic $p>0$ situation.

Proposition 2.1. Let $\operatorname{char} K=p>0$ and let e be an idempotent in $K^{t}[G]$. Assume that $\mathfrak{K}_{y}$ is consistent for all special elements $y \in \operatorname{supp} e$. If $\mathfrak{K}_{g}$ is consistent and if $\operatorname{tr}_{\bar{g}} e \neq 0$, then there exists a positive integer $n \leq|\operatorname{supp} e|$ such that $g^{p^{n}}$ is conjugate to $g$, and such that

$$
\operatorname{tr}_{\bar{g}} e=\operatorname{tr}_{\bar{g}} \bar{g}^{p^{n}} \cdot\left(\operatorname{tr}_{\bar{g}} e\right)^{p^{n}} .
$$

Furthermore, there exists a special element $h \in \operatorname{supp} e$ conjugate to $g^{p}$ with

$$
\operatorname{tr}_{\bar{h}} e=\operatorname{tr}_{\bar{h}} \bar{g}^{p} \cdot\left(\operatorname{tr}_{\bar{g}} e\right)^{p} .
$$

Proof. Let $S=\operatorname{supp} e$ and let $\mathcal{K}=\left\{\mathfrak{K}_{g_{1}}, \mathfrak{K}_{g_{2}}, \ldots, \mathfrak{K}_{g_{r}}\right\}$ denote the finitely many consistent conjugacy classes of $G$ that satisfy $\operatorname{tr}_{\bar{g}_{i}} e \neq 0$. By assumption, $\mathfrak{K}_{g} \in \mathcal{K}$. Furthermore, $\operatorname{tr}_{\bar{g}_{i}} e \neq 0$ implies that $\mathfrak{K}_{g_{i}} \cap S \neq \emptyset$, so $r=|\mathcal{K}| \leq|S|$. For simplicity, let us write $\mathfrak{K}_{i}$ for $\mathfrak{K}_{g_{i}}$ and $\operatorname{tr}_{i}$ for $\operatorname{tr}_{\bar{g}_{i}}$.

Let $e=\sum_{x \in S} a_{x} \bar{x}$. Since $\operatorname{char} K=p$, we have $e=e^{p}=\sum_{x \in S} a_{x}^{p} \bar{x}^{p}+\beta$ for some element $\beta \in\left[K^{t}[G], K^{t}[G]\right]$. Now fix a subscript $i$ and note that

$$
0 \neq \operatorname{tr}_{i} e=\operatorname{tr}_{i} e^{p}=\sum_{x \in S} a_{x}^{p} \cdot \operatorname{tr}_{i} \bar{x}^{p}=\sum_{x^{p} \sim g_{i}} a_{x}^{p} \cdot \operatorname{tr}_{i} \bar{x}^{p},
$$

since $\operatorname{tr}_{i} \beta=0$. In particular, there exists $y \in S$ with $y^{p} \sim g_{i}$ and, by Lemma 1.1(ii) and our hypothesis, we know that each such $\mathfrak{K}_{y}$ is special and consistent. Furthermore, if $z \in G$ is conjugate to $y$, then $\bar{z}$ is conjugate to $\bar{y}$ by consistency, and hence $\bar{z}^{p}$ is conjugate to $\bar{y}^{p}$. Thus, since $\operatorname{tr}_{i}$ is a trace map, it follows that $\operatorname{tr}_{i} \bar{z}^{p}$ is constant for all $z \in \mathfrak{K}_{y}$.

If $y_{1}, y_{2}, \ldots \in S$ are representatives of the conjugacy classes with $y_{j}^{p} \sim g_{i}$, then the above displayed equation yields

$$
\begin{aligned}
0 \neq \operatorname{tr}_{i} e & =\sum_{j} \operatorname{tr}_{i}{\overline{y_{j}}}^{p} \cdot \sum_{x \sim y_{j}} a_{x}^{p} \\
& =\sum_{j} \operatorname{tr}_{i}{\overline{y_{j}}}^{p} \cdot\left(\sum_{x \sim y_{j}} a_{x}\right)^{p}=\sum_{j} \operatorname{tr}_{i}{\overline{y_{j}}}^{p} \cdot\left(\operatorname{tr}_{\overline{y_{j}}} e\right)^{p} .
\end{aligned}
$$

We can, of course, delete those terms in the above sum with $\operatorname{tr}_{\overline{y_{j}}} e=0$, and after doing this, the remaining $y_{j}$ are then representatives of conjugacy classes in $\mathcal{K}$. To be specific, let $\mathcal{K}_{i} \subseteq \mathcal{K}$ denote the set of all the conjugacy classes for the various remaining $y_{j}$. Then we have

$$
0 \neq \operatorname{tr}_{i} e=\sum_{\mathfrak{K}_{y} \in \mathcal{K}_{i}} \operatorname{tr}_{i} \bar{y}^{p} \cdot\left(\operatorname{tr}_{\bar{y}} e\right)^{p} .
$$

In other words, each $i$ gives rise to a nonempty subset $\mathcal{K}_{i}$ of $\mathcal{K}$, and these subsets are disjoint since if $\mathfrak{K}_{y} \in \mathcal{K}_{i} \cap \mathcal{K}_{j}$, then $g_{i} \sim y^{p} \sim g_{j}$ and $i=j$. Thus, we have $r=|\mathcal{K}|$ nonempty disjoint subsets of $\mathcal{K}$, and this clearly implies that each $\mathcal{K}_{i}$ is a singleton and that their union is $\mathcal{K}$. In particular, we can now define a map $\sigma$ that sends $\mathfrak{K}_{i}$ 
to the unique conjugacy class $\mathfrak{K}_{\nu(i)}$ in $\mathcal{K}_{i}$. Then $\sigma$ is clearly a permutation of $\mathcal{K}$, with $\nu$ the corresponding permutation of the subscripts $1,2, \ldots, r$. Furthermore, for all $i=1,2, \ldots, r$, we have

$$
g_{\nu(i)}^{p} \sim g_{i} \quad \text { and } \quad \operatorname{tr}_{i} e=\operatorname{tr}_{i}{\overline{g_{\nu(i)}}}^{p} \cdot\left(\operatorname{tr}_{\nu(i)} e\right)^{p} .
$$

Recall that $\mathfrak{K}_{g} \in \mathcal{K}$, and let $n \leq|\mathcal{K}| \leq|S|$ be the length of the cycle of $\sigma$ that contains this element. We can, of course, assume the numbering so chosen that $\mathfrak{K}_{g}$ corresponds to $i=1$ and that $\nu$ contains the $n$-cycle $\left(\begin{array}{llll}1 & 2 \cdots n\end{array}\right)$. We show by induction on $i=1,2, \ldots, n$ that

$$
g_{\nu(i)}^{p^{i}} \sim g \quad \text { and } \quad \operatorname{tr}_{\bar{g}} e=\operatorname{tr}_{\bar{g}}{\overline{g_{\nu(i)}}}^{i} \cdot\left(\operatorname{tr}_{\nu(i)} e\right)^{p^{i}}
$$

Since $\mathfrak{K}_{g}=\mathfrak{K}_{1}$, this is equation (因) when $i=1$. Now suppose the result holds for $i<n$. Then $\nu(i) \leq n$ and $\nu(\nu(i))=\nu(i+1)$, so (丑), with $i$ replaced by $\nu(i)$, yields $\operatorname{tr}_{\nu(i)} e=\operatorname{tr}_{\nu(i)}{\overline{g_{\nu(i+1)}}}^{p} \cdot\left(\operatorname{tr}_{\nu(i+1)} e\right)^{p}$ and hence

$$
\begin{aligned}
\operatorname{tr}_{\bar{g}} e & =\operatorname{tr}_{\bar{g}}{\overline{g_{\nu(i)}}}^{p^{i}} \cdot\left(\operatorname{tr}_{\nu(i)} e\right)^{p^{i}} \\
& =\operatorname{tr}_{\bar{g}}{\overline{g_{\nu(i)}}}^{i} \cdot\left(\operatorname{tr}_{\nu(i)} \overline{g_{\nu(i+1)}}\right)^{p^{i}} \cdot\left(\operatorname{tr}_{\nu(i+1)} e\right)^{p^{i+1}}
\end{aligned}
$$

Furthermore, by induction, $\overline{g_{\nu(i)}} p^{i}=\eta \bar{g}^{x}$ for some $\eta \in K^{\bullet}, x \in G$, and we know, from (函), that ${\overline{g_{\nu(i+1)}}}^{p}=\mu{\overline{g_{\nu(i)}}}^{y}$ for some $\mu \in K^{\bullet}, y \in G$. Thus

$$
\overline{g_{\nu(i+1)}} p^{i+1}=\mu^{p^{i}}\left({\overline{g_{\nu(i)}}}_{p^{i}}\right)^{y}=\eta \mu^{p^{i}} \bar{g}^{x y} .
$$

In particular, we have $g_{\nu(i+1)}^{p^{i+1}} \sim g$ and

$$
\operatorname{tr}_{\bar{g}}{\overline{g_{\nu(i+1)}}}^{i+1}=\eta \mu^{p^{i}}=\operatorname{tr}_{\bar{g}}{\overline{g_{\nu(i)}}}^{p^{i}} \cdot\left(\operatorname{tr}_{\nu(i)}{\overline{g_{\nu(i+1)}}}^{p}\right)^{p^{i}}
$$

so it follows that $\operatorname{tr}_{\bar{g}} e=\operatorname{tr}_{\bar{g}}{\overline{g_{\nu(i+1)}}}^{p^{i+1}} \cdot\left(\operatorname{tr}_{\nu(i+1)} e\right)^{p^{i+1}}$, as required.

Finally, when $i=n$, we have $\nu(n)=1$ so $g^{p^{n}} \sim g$ and $\operatorname{tr}_{\bar{g}} e=\operatorname{tr}_{\bar{g}} \bar{g}^{p^{n}} \cdot\left(\operatorname{tr}_{\bar{g}} e\right)^{p^{n}}$. This proves the first part of the proposition. For the second part, set $h=g_{n}$. Since $\sigma\left(\mathfrak{K}_{h}\right)=\sigma\left(\mathfrak{K}_{n}\right)=\mathfrak{K}_{1}=\mathfrak{K}_{g}$, it therefore follows from (図) that $g^{p} \sim h$ and also that $\operatorname{tr}_{\bar{h}} e=\operatorname{tr}_{\bar{h}} \bar{g}^{p} \cdot\left(\operatorname{tr}_{\bar{g}} e\right)^{p}$.

As a consequence, we have

Corollary 2.2. Let char $K=p>0$ and let $e$ be an idempotent in $K^{t}[G]$. Assume that $\mathfrak{K}_{g}$ is consistent and normalized and that $\operatorname{tr}_{\bar{g}} e \neq 0$. Then there exists a positive integer $n \leq|\operatorname{supp} e|$ such that $g^{p^{n}}$ is conjugate to $g$ and such that

$$
\operatorname{tr}_{\bar{g}} e=\lambda\left(\operatorname{tr}_{\bar{g}} e\right)^{p^{n}}
$$

where $\lambda \in K^{\bullet}$ is a $c(g)^{\text {th }}$ root of unity. In particular, $\operatorname{tr}_{\bar{g}}$ e is algebraic over $\mathrm{GF}(p)$.

Proof. We can assume that if $h \in \operatorname{supp} e=S$ and $h$ is special, then $\mathfrak{K}_{h}$ is consistent. Thus, by Proposition 2.1 $g^{p^{n}}$ is conjugate to $g$ for some positive integer $n \leq|S|$

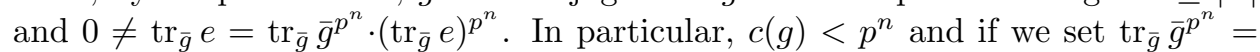
$\lambda \in K^{\bullet}$, then $\bar{g}^{p^{n}}=\lambda \bar{g}^{z}$ for some $z \in G$. But $\mathfrak{K}_{g}$ is normalized and consistent, so Lemma 1.5(ii) implies that $\lambda$ is a $c(g)^{\text {th }}$ root of unity. 
Next, we turn to fields $K$ of characteristic 0 , and we begin with

Proposition 2.3. Let char $K=0$ and let e be an idempotent of $K^{t}[G]$. If $\mathfrak{K}_{g}$ is a consistent, normalized conjugacy class, then $\operatorname{tr}_{\bar{g}}$ e is algebraic over the rational field $Q$. Furthermore, if $\operatorname{tr}_{\bar{g}} e \neq 0$, then there exists a cofinite subset $\mathcal{P}$ of the set of rational primes such that, for each $p \in \mathcal{P}$, we have $g^{p^{n}} \sim g$ for some positive integer $n=n(p) \leq|\operatorname{supp} e|$ and we have an element $h=h(p) \in \operatorname{supp} e$ with $h \sim g^{p}$. In particular, $\operatorname{tr}_{\bar{g}} e \neq 0$ implies that $c(g)<\infty$.

Proof. If $f(\zeta)=\zeta^{2}-\zeta \in K[\zeta]$, then $f(e)=0$ and, in view of Lemma 1.6, we can assume that $G$ is finitely presented. Then, by Lemma 1.7 applied to the class $\mathfrak{K}_{g}$, we can assume that $\tau(G, G)$ is contained in a finitely generated subgroup $T$ of $K^{\bullet}$. If $e=\sum a_{x} \bar{x}$, then we let $R$ be the subring of $K$ generated over the integers $Z$ by the finitely many $a_{x}$ with $x \in \operatorname{supp} e$ and by the finitely many generators of $T$ and their inverses. It follows that $\tau(G, G)$ is a subset of the units of $R$ and that $e \in R^{t}[G]$. We may clearly suppose that $\operatorname{tr}_{\bar{g}} e \neq 0$.

If $\operatorname{tr}_{\bar{g}} e$ is transcendental over $Q$, then it follows from [5, Theorem 1.1.9] that there exists a ring homomorphism $\theta: R \rightarrow F$, with $F$ an algebraically closed field of characteristic $p>0$, and with $\theta\left(\operatorname{tr}_{\bar{g}} e\right)$ transcendental over $\operatorname{GF}(p)$. Furthermore, by Lemma 1.8, there exists a twisted group algebra $F^{t}[G]$ and a homomorphism $\theta: R^{t}[G] \rightarrow F^{t}[G]$ given by $\sum r_{x} \bar{x} \mapsto \sum \theta\left(r_{x}\right) \bar{x}$. In particular, $\theta(e)$ is an idempotent of $F^{t}[G]$ with $\operatorname{tr}_{\bar{g}} \theta(e)=\theta\left(\operatorname{tr}_{\bar{g}} e\right)$ transcendental over $\operatorname{GF}(p)$. But $\mathfrak{K}_{g}$ is a consistent, normalized class in $F^{t}[G]$, and therefore this contradicts Corollary [2.2 $\operatorname{Thus} \operatorname{tr}_{\bar{g}} e$ must be algebraic over $Q$.

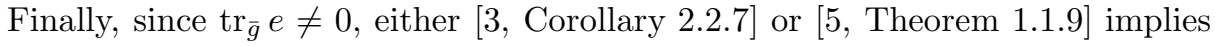
that there exists a cofinite subset $\mathcal{P}$ of the set of rational primes such that, for each $p \in \mathcal{P}$, there is a ring homomorphism $\theta_{p}: R \rightarrow F_{p}$, with $F_{p}$ an algebraically closed field of characteristic $p$ and with $\theta\left(\operatorname{tr}_{\bar{g}} e\right) \neq 0$. (Both of the above-cited results speak about infinite sets of primes, but their proofs show that only finitely many primes need to be avoided.) Again, Lemma 1.8 yields twisted group algebras $F_{p}^{t}[G]$ and extended homomorphisms $\theta_{p}: R^{t}[G] \rightarrow F_{p}^{t}[G]$ given by $\sum r_{x} \bar{x} \mapsto \sum \theta_{p}\left(r_{x}\right) \bar{x}$. Thus $\theta_{p}(e)$ is an idempotent of $F_{p}^{t}[G]$ with $\operatorname{supp} \theta_{p}(e) \subseteq \operatorname{supp} e . \operatorname{Since}_{\operatorname{tr}_{\bar{g}}} \theta_{p}(e)=$ $\theta_{p}\left(\operatorname{tr}_{\bar{g}} e\right) \neq 0$ and since $\mathfrak{K}_{g}$ is consistent and normalized in $F_{p}^{t}[G]$, Proposition 2.1 implies that $g^{p^{n}} \sim g$ for some positive integer $n=n(p) \leq\left|\operatorname{supp} \theta_{p}(e)\right| \leq|\operatorname{supp} e|$. In addition, there exists $h=h(p) \in \operatorname{supp} e$ with $h \sim g^{p}$.

Finally, we obtain our main result, the twisted analog of [1 Theorem 8.1]. As in the original argument, we require both the Frobenius Density Theorem and the Kronecker-Weber Theorem.

Theorem 2.4. Let char $K=0$ and let $e$ be an idempotent of the twisted group algebra $K^{t}[G]$. If $\mathfrak{K}_{g}$ is a consistent, normalized conjugacy class of the group $G$, then $\operatorname{tr}_{\bar{g}}$ e is contained in a cyclotomic field.

Proof. If $f(\zeta)=\zeta^{2}-\zeta \in K[\zeta]$, then $f(e)=0$ and hence, by Lemma 1.6 we can assume that $G$ is finitely presented. Furthermore, by Lemma 1.7 applied to the class $\mathfrak{K}_{g}$, we can assume that $\tau(G, G)$ is contained in a finitely generated subgroup $T_{0}$ of $K^{\bullet}$. If $e=\sum a_{x} \bar{x}$, then we let $R_{0}$ be the subring of $K$ generated over the integers $Z$ by the finitely many $a_{x}$ with $x \in \operatorname{supp} e$ and by the finitely many 
generators of $T_{0}$ and their inverses. It follows that $\tau(G, G)$ is a subset of the units of $R_{0}$, and that $e \in R_{0}^{t}[G]$. We may clearly suppose that $\operatorname{tr}_{\bar{g}} e \neq 0$ and, by Proposition [2.3, we know that $\operatorname{tr}_{\bar{g}} e$ is algebraic over the rational field $Q$ and that $c=c(g)<\infty$. If $\widetilde{Q}$ denotes the algebraic closure of $Q$, then it follows from [3. Corollary 2.2.6] that there exists a homomorphism $\kappa: R_{0} \rightarrow \widetilde{Q}$ which is the identity map on $R_{0} \cap \widetilde{Q}$. By Lemma [1.8, there exists a twisted group algebra $\widetilde{Q}^{t}[G]$ and an extended homomorphism $\kappa: R_{0}^{t}[G] \rightarrow \widetilde{Q}^{t}[G]$ with $\operatorname{tr}_{\bar{g}} \kappa(e)=\kappa\left(\operatorname{tr}_{\bar{g}} e\right)=\operatorname{tr}_{\bar{g}} e$. In other words, we may now assume that $G$ is finitely presented and that $K=\widetilde{Q}$.

By Lemma 1.4 we can suppose that, for every special element $h \in \operatorname{supp} e$, the conjugacy class $\mathfrak{K}_{h}$ is consistent and normalized. Furthermore, by Lemma 1.7 applied to $\mathfrak{K}_{g}$ and these finitely many classes, we may again suppose that $\tau(G, G)$ is contained in a finitely generated subgroup $T$ of $\widetilde{Q}$. If $e=\sum a_{x} \bar{x}$, then we let $S^{\prime}$ be the subring of $\widetilde{Q}$ generated over the integers $Z$ by the finitely many $a_{x}$ with $x \in \operatorname{supp} e$ and by the finitely many generators of $T$ and their inverses. For convenience, we also assume that $S^{\prime}$ contains a primitive $c^{\text {th }} \operatorname{root}$ of unity. Then $S^{\prime}$ is contained in a finite normal extension field $L$ of $Q$ with Galois group $\mathcal{G}=\operatorname{Gal}(L / Q)$. Since $\mathcal{G}$ is finite, the subring $S$ of $L$ generated by $S^{\prime}$ and all it $\mathcal{G}$-conjugates is also finitely generated. Thus $S$ is a finitely generated $\mathcal{G}$-stable subring of $L, \tau(G, G)$ is a subset of the units of $S$, and $e \in S^{t}[G]$.

Next, we adjoin to $S$ the element $\left(\operatorname{tr}_{\bar{g}} e\right)^{-1}$ and its finitely many $\mathcal{G}$-conjugates. Furthermore, for each of the finitely many special elements $h \in \operatorname{supp} e$, for each of the finitely many pairs $\sigma_{1}, \sigma_{2} \in \mathcal{G}$, and for each of the finitely many $c^{\text {th }}$ roots of unity $\lambda \in S$, if $\sigma_{1}\left(\operatorname{tr}_{\bar{h}} e\right)-\lambda \cdot \sigma_{2}\left(\operatorname{tr}_{\bar{g}} e\right) \neq 0$, then we adjoin to $S$ the inverse of this element. In this way, we obtain a finitely generated $\mathcal{G}$-stable $\operatorname{ring} R$ with $S \subseteq R \subseteq L$ and with $e \in R^{t}[G]$. Our goal now is to understand the action of $\mathcal{G}$ on the normal closure of $\operatorname{tr}_{\bar{g}} e$, namely the subfield $E$ of $L$ generated by all $\mathcal{G}$-conjugates of $\operatorname{tr}_{\bar{g}} e$. To this end, let $\mathcal{C}$ be an arbitrary cyclic subgroup of $\mathcal{G}$. Then the Frobenius Density Theorem (see [2. Theorem IV.5.2]) implies that there is a generator $\eta$ of $\mathcal{C}$ and an infinite family $\mathcal{Q}^{\prime}$ of rationals primes such that, for each $p \in \mathcal{Q}^{\prime}$, there exists a homomorphism $\theta_{p}: R \rightarrow F_{p}$, where $F_{p}$ is an algebraically closed field of characteristic $p$ and where $\theta_{p}(\eta(r))=\theta_{p}(r)^{p}$ for all $r \in R$.

Let $\mathcal{P}$ be the subset of rational primes given by Proposition 2.3 Then $\mathcal{P}$ is cofinite and $\mathcal{Q}^{\prime}$ is infinite, so $\mathcal{P} \cap \mathcal{Q}^{\prime}$ is also infinite. By definition of $\mathcal{P}$, for each $p \in \mathcal{P} \cap \mathcal{Q}^{\prime}$, there exists an element $h(p) \in \operatorname{supp} e$ with $h(p) \sim g^{p}$. Furthermore, $g^{p^{n(p)}} \sim g$ for some positive integer $n(p) \leq|\operatorname{supp} e|$. Since $\mathcal{P} \cap \mathcal{Q}^{\prime}$ is infinite and supp $e$ is finite, there is an infinite subset $\mathcal{Q}$ of $\mathcal{P} \cap \mathcal{Q}^{\prime}$, a fixed element $h \in \operatorname{supp} e$ and a fixed positive integer $n \leq|\operatorname{supp} e|$, with $h(p)=h$ and $n(p)=n$ for all $p \in \mathcal{Q}$. Observe that if $p$ is in $\mathcal{Q}$, then $g^{p} \sim h$ and $g^{p^{n}} \sim g$, so $h^{p^{n-1}} \sim g$. In particular, this formula holds for two different primes $p_{1}, p_{2} \in \mathcal{Q}$ and, since $p_{1}^{n-1}$ and $p_{2}^{n-1}$ are relatively prime, we conclude from Lemma 1.1(i) that $h$ is special. By our assumptions, $\mathfrak{K}_{h}$ is therefore a consistent and normalized conjugacy class.

At this point, we fix a prime $p$ in $\mathcal{Q}$, and we let $\theta: R \rightarrow F$ denote the corresponding homomorphism to an algebraically closed field $F$ of characteristic $p$. Of course, $\theta(\eta(r))=\theta(r)^{p}$ for all elements $r \in R$. Note that $g^{p} \sim h$ and $h^{p^{n-1}} \sim g$, so Lemma 1.5(i) implies that $c(h)=c(g)=c$. Now let $\xi \in \mathcal{G}$. Then, by Lemma 1.8 the combined map

$$
\theta \xi: R \stackrel{\xi}{\longrightarrow} R \stackrel{\theta}{\rightarrow} F
$$


determines a twisted group algebra over $F$, which we denote by $F_{\xi}^{t}[G]$, and an extended homomorphism $\theta \xi: R^{t}[G] \rightarrow F_{\xi}^{t}[G]$ given by $\sum r_{x} \bar{x} \mapsto \sum \theta \xi\left(r_{x}\right) \bar{x}$. In particular, $\theta \xi(e)$ is an idempotent in $F_{\xi}^{t}[G]$ with $\operatorname{supp} \theta \xi(e) \subseteq \operatorname{supp} e$. Furthermore, we know that both $\mathfrak{K}_{g}$ and $\mathfrak{K}_{h}$ are consistent and normalized in $F_{\xi}^{t}[G]$ and that, for all $\alpha \in R^{t}[G]$, we have $\operatorname{tr}_{\bar{g}} \theta \xi(\alpha)=\theta \xi\left(\operatorname{tr}_{\bar{g}} \alpha\right)$ and $\operatorname{tr}_{\bar{h}} \theta \xi(\alpha)=\theta \xi\left(\operatorname{tr}_{\bar{h}} \alpha\right)$.

Now $\theta \xi(e)=\sum \theta \xi\left(a_{x}\right) \bar{x}$ is an idempotent in $F_{\xi}^{t}[G]$ and, via a diagonal change of basis, we can make consistent all conjugacy classes of elements of $\operatorname{supp} \theta \xi(e)$ which have become special. Since $\operatorname{tr}_{\bar{g}} e$ is a unit in $R$, it follows that $\operatorname{tr}_{\bar{g}} \theta \xi(e) \neq 0$ and then, since $h \sim g^{p}$, Proposition 2.1 implies that

$$
\begin{aligned}
\theta \xi\left(\operatorname{tr}_{\bar{h}} e\right) & =\operatorname{tr}_{\bar{h}} \theta \xi(e)=\operatorname{tr}_{\bar{h}} \theta \xi(\bar{g})^{p} \cdot\left(\operatorname{tr}_{\bar{g}} \theta \xi(e)\right)^{p}=\operatorname{tr}_{\bar{h}} \theta \xi\left(\bar{g}^{p}\right) \cdot \theta \xi\left(\operatorname{tr}_{\bar{g}} e\right)^{p} \\
& =\theta \xi\left(\operatorname{tr}_{\bar{h}} \bar{g}^{p}\right) \cdot \theta\left(\xi\left(\operatorname{tr}_{\bar{g}} e\right)^{p}\right)=\theta \xi\left(\operatorname{tr}_{\bar{h}} \bar{g}^{p}\right) \cdot \theta\left(\eta \xi\left(\operatorname{tr}_{\bar{g}} e\right)\right),
\end{aligned}
$$

since $\theta(r)^{p}=\theta \eta(r)$ for all $r \in R$. In other words, $\xi\left(\operatorname{tr}_{\bar{h}} e\right)-\xi\left(\operatorname{tr}_{\bar{h}} \bar{g}^{p}\right) \cdot \eta \xi\left(\operatorname{tr}_{\bar{g}} e\right)$ is an element of $R$ that maps to 0 under $\theta$. Furthermore, we know that $\xi\left(\operatorname{tr}_{\bar{h}} \bar{g}^{p}\right)=\lambda$ is a $c^{\text {th }}$ root of unity by Lemma 1.5(i). Since $\xi\left(\operatorname{tr}_{\bar{h}} e\right)-\lambda \cdot \eta \xi\left(\operatorname{tr}_{\bar{g}} e\right)$ is not a unit in $R$, this element must be 0 , by the definition of $R$.

We have therefore shown that

$$
\xi\left(\operatorname{tr}_{\bar{h}} e\right)=\xi\left(\operatorname{tr}_{\bar{h}} \bar{g}^{p}\right) \cdot \eta \xi\left(\operatorname{tr}_{\bar{g}} e\right) \quad \text { for all } \xi \in \mathcal{G} .
$$

In particular, if we fix $\sigma \in \mathcal{G}$ and replace $\xi$ by $\xi \sigma$ in (玦), then we have

$$
\xi \sigma\left(\operatorname{tr}_{\bar{h}} e\right)=\xi \sigma\left(\operatorname{tr}_{\bar{h}} \bar{g}^{p}\right) \cdot \eta \xi\left(\sigma\left(\operatorname{tr}_{\bar{g}} e\right)\right) .
$$

On the other hand, if we replace $\xi$ by $\sigma$ in (沵) and then apply the automorphism $\xi$ to the resulting equation, we get

$$
\xi \sigma\left(\operatorname{tr}_{\bar{h}} e\right)=\xi \sigma\left(\operatorname{tr}_{\bar{h}} \bar{g}^{p}\right) \cdot \xi \eta\left(\sigma\left(\operatorname{tr}_{\bar{g}} e\right)\right) .
$$

The preceding two displayed equations now yield

$$
\eta \xi\left(\sigma\left(\operatorname{tr}_{\bar{g}} e\right)\right)=\xi \eta\left(\sigma\left(\operatorname{tr}_{\bar{g}} e\right)\right)
$$

for all $\xi, \sigma \in \mathcal{G}$. This shows that $\eta$ and $\xi$ commute when restricted to $E$, the normal closure of $\operatorname{tr}_{\bar{g}} e$, and hence, since $\operatorname{res}_{E} \mathcal{G}=\operatorname{Gal}(E / Q)$, the restriction of $\eta$ is central in $\operatorname{Gal}(E / Q)$. But $\mathcal{C}=\langle\eta\rangle$ is an arbitrary cyclic subgroup of $\mathcal{G}$, so the restriction of $\mathcal{G}$ to $E$ is abelian, and consequently $\operatorname{res}_{E} \mathcal{G}=\operatorname{Gal}(E / Q)$ is abelian. It now follows from the Kronecker-Weber Theorem (see [2, Theorem V.5.10]) that $E$ is contained in a cyclotomic field and, since $\operatorname{tr}_{\bar{g}} e \in E$, the theorem is proved.

In the above, if $\operatorname{tr}_{\bar{g}} e \in Q[\epsilon]$ with $\epsilon^{k}=1$, then one expects the exponent $k$ to be related to the orders of the torsion elements of $G$. A precise conjecture of this nature, in the case of ordinary group algebras, is proposed in [1].

\section{REFERENCES}

[1] H. Bass, Euler characteristics and characters of discrete groups, Invent. Math. 35 (1976), 155-196. MR 55:5764

[2] G. J. Janusz, Algebraic Number Fields, $2^{\text {nd }}$ edition, American Math. Soc., Providence, 1996. MR 96j:11137

[3] D. S. Passman, The Algebraic Structure of Group Rings, Wiley-Interscience, New York, 1977. MR 81d:16001; reprint MR 86j:16001 
[4] D. S. Passman, Trace methods in twisted group algebras, Proc. AMS 129 (2001), 943-946. MR 2001g:16054

[5] S. K. Sehgal, Topics in Group Rings, Marcel Dekker, New York, 1978. MR 80j:16001

Department of Mathematics, University of Cincinnati, Cincinnati, Ohio 45221

E-mail address: james.osterburg@math.uc.edu

Mathematics Department, University of Wisconsin, Madison, Wisconsin 53706

E-mail address: passman@math.wisc.edu 